\title{
Creative Computing and Computational Aesthetics
}

Nereida Rodríguez-Fernández, Iria Santos, Álvaro Torrente-Patiño, Luz Castro Pena

Albert Szent-Györgyi defined a creative act as 'seeing what everyone else has seen and thinking as no one else has thought'. Would a machine be able to perform a creative act? Ada Lovelace, considered the first woman programmer, proposed almost 200 years ago the use of computers for artistic creative tasks, specifically musical ones.

In recent years, our research group has been working on the creation of artificial artists and critics, as well as on classifiers and predictors of complexity and aesthetics.

Automatic prediction of the aesthetic value of images has received increasing attention in recent years. This has come about, in part, because of the potential impact that aesthetic value has on practical applications.

An aesthetic image evaluation system has been developed in recent years by our research group and has recently been tested for its potential and effectiveness in practical applications for commercial tasks. The results suggest an increase in the impact of advertisements using aesthetic criteria. 